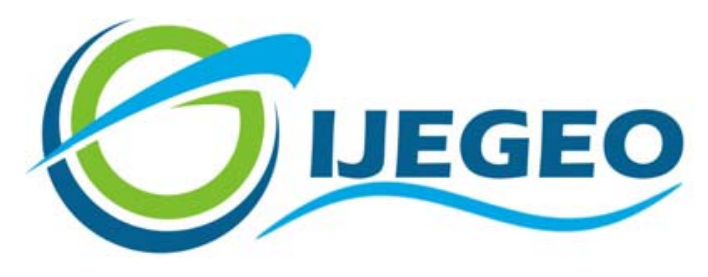

International Journal of Environment and Geoinformatics (IJEGEO) is an international, multidisciplinary, peer reviewed, open access journal.

\title{
Unmixing of Hyperspectral Data Using Spectral Libraries
}

\author{
Sefa Küçük, Seniha Esen Yüksel
}

\section{Chief in Editor}

Prof. Dr. Cem Gazioğlu

\section{Co-Editor}

Prof. Dr. Dursun Zafer Şeker, Prof. Dr. Şinasi Kaya,

Prof. Dr. Ayşegül Tanık and Assist. Prof. Dr. Volkan Demir

Editorial Committee (April 2020)

Assos. Prof. Dr. Abdullah Aksu (TR), Assit. Prof. Dr. Uğur Algancı (TR), Prof. Dr. Bedri Alpar (TR), Prof. Dr. Lale Balas (TR), Prof. Dr. Levent Bat (TR), Prof. Dr. Paul Bates (UK), İrşad Bayırhan (TR), Prof. Dr. Bülent Bayram (TR), Prof. Dr. Luis M. Botana (ES), Prof. Dr. Nuray Çağlar (TR), Prof. Dr. Sukanta Dash (IN), Dr. Soofia T. Elias (UK), Prof. Dr. A. Evren Erginal (TR), Assoc. Prof. Dr. Cüneyt Erenoğlu (TR), Dr. Dieter Fritsch (DE), Assos. Prof. Dr. Çiğdem Göksel (TR), Prof.Dr. Lena Halounova (CZ), Prof. Dr. Manik Kalubarme (IN), Dr. Hakan Kaya (TR), Assist. Prof. Dr. Serkan Kükrer (TR), Assoc. Prof. Dr. Maged Marghany (MY), Prof. Dr. Michael Meadows (ZA), Prof. Dr. Nebiye Musaoğlu (TR), Prof. Dr. Erhan Mutlu (TR), Prof. Dr. Masafumi Nakagawa (JP), Prof. Dr. Hasan Özdemir (TR), Prof. Dr. Chryssy Potsiou (GR), Prof. Dr. Erol Sarı (TR), Prof. Dr. Maria Paradiso (IT), Prof. Dr. Petros Patias (GR), Prof. Dr. Elif Sertel (TR), Prof. Dr. Nüket Sivri (TR), Prof. Dr. Füsun Balık Şanlı (TR), Prof. Dr. Uğur Şanlı (TR), Duygu Ülker (TR), Assoc. Prof. Dr. Oral Yağcı (TR), Prof. Dr. Seyfettin Taş (TR), Assoc. Prof. Dr. Ömer Suat Taşkın (US), Dr. İnese Varna (LV), Dr. Petra Visser (NL), Prof. Dr. Selma Ünlü (TR), Assoc. Prof. Dr. İ. Noyan Yilmaz (AU), Prof. Dr. Murat Yakar (TR), Assit. Prof. Dr. Sibel Zeki (TR) 


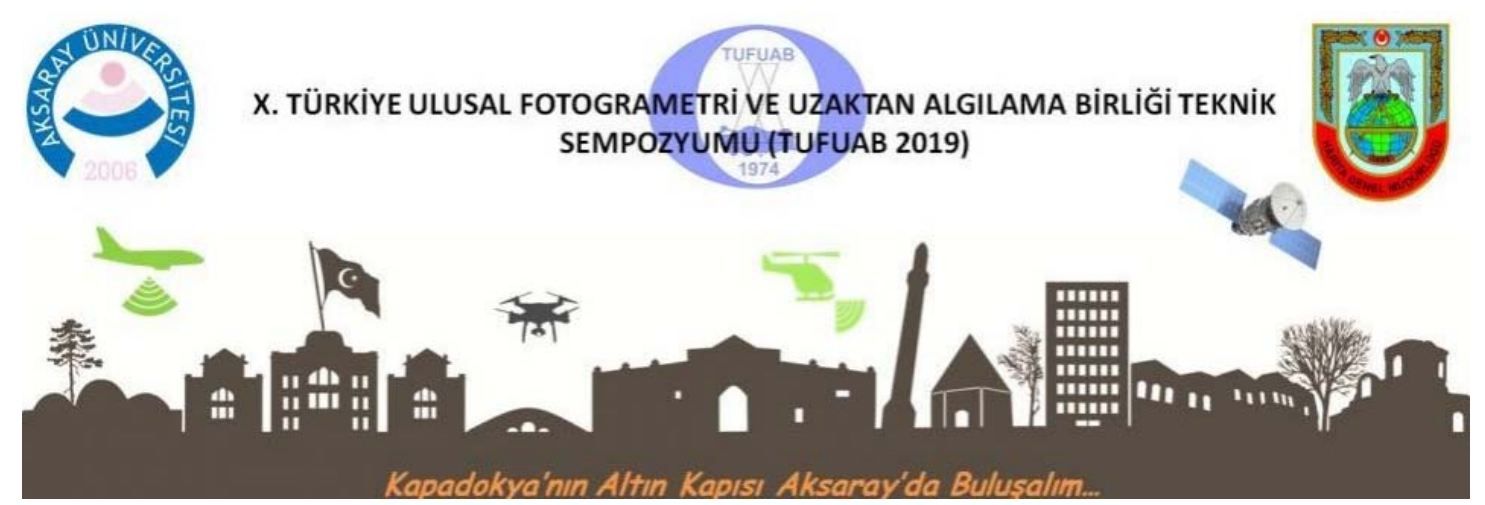

Dear colleagues and friends,

X. TUFUAB Technical Symposium was held in Aksaray on 25-27, April 2019. The Symposium was carried out by the organizations of Aksaray University and General Directorate of Mapping. As a international symposium in the field of photogrammetry and remote sensing, X.TUFUAB Technical Symposium 2019 is devoted to promote the advancement of knowledge, research, development, education and training in Geographical Information Sciences, Information Technology, Environmental Management and Resources, Sustainable Agriculture, Surveying, Photogrammetry and Remote Sensing, their integration and applications, as to contribute to the well-being of humanity and the sustainability of the environment. 425 participants and scientists from 7 countries were attended to this symposium. 125 oral presentations and 10 poster presentations were presented during the symposium. 135 presentations take place in 25 sessions in two days.

The presentations were reviewed by the scientific committee. Nine of these presentations were found worthy to be published in "International Journal of Environment and Geoinformatics (IJEGEO)" by the scientific committee. We would like to thank editorial board of IJEGEO for the publication of these works in the symposium.

Aksaray, Turkey, April 2019.

Prof. Dr. H. Murat YILMAZ

Chair of the Organizing Committee
Dr. Eng. Tuğg. Oman ALP

Director, General Directorate of Mapping 


\title{
Unmixing of Hyperspectral Data Using Spectral Libraries
}

\author{
Sefa Küçük* $^{*}$, Seniha Esen Yüksel (iD) \\ ${ }^{1}$ Electrical and Electronics Engineering, Faculty of Engineering, Hacettepe University, Ankara-TR \\ *Corresponding author: S. Kucuk \\ E-mail:skucuk@ee.hacettepe.edu.tr
}

Received 21 Nov 2019

Accepted 27 March 2020

How to cite: Kucuk and Yuksel (2020). Unmixing of Hyperspectral Data Using Spectral Libraries, International Journal of Environment and Geoinformatics (IJEGEO), 7(1):93-101, DOI: 10.30897/ijegeo.649394

\begin{abstract}
In hyperspectral images, pixels are found as a mixture of the spectral signatures of several materials, especially when there is an insufficient spatial resolution. In recent years, spectral libraries have provided spectral information of hundreds of materials that allow the development of techniques to solve the problem of hyperspectral unmixing in a semi-supervised fashion. These methods which are also known as sparse regression techniques assume that mixed pixels are a sparse linear combination of spectral signatures of materials in already available spectral libraries. In this paper, the spectral mixing problem has been solved via sparse separation methods. The United States Geological Survey (USGS) spectral library is used to generate simulated hyperspectral data. A comparative analysis is performed to determine which material signatures in the library are mixed in the pixels by using the convexrelaxation-based sparse regression methods. Root Mean Square Error (RMSE), Signal to Reconstruction Error (SRE) and processing time of the algorithms are used as comparing criterions. Moreover, Hinton diagrams are used to visualize which material signatures are found in the library and the proportions of these found material signatures.
\end{abstract}

Keywords: Hyperspectral Imaging, Hyperspectral Unmixing, Sparse Regression, Sparse Unmixing, Spectral Library

\section{Introduction}

Remote sensing is a technology based on the measurement of reflected or radiated energy from surface materials. Hyperspectral imaging is one of the technologies that can measure the spectra of the Earth's surface in hundreds of narrow and adjacent wavelength bands. Hyperspectral images are obtained using devices that measure reflected or radiated energy from materials with respect to wavelengths. The spectral information of materials at different wavelengths creates the unique spectral patterns so-called spectral signatures which allow identification and classification of the materials (Kucuk, 2015). Over the last decades, hyperspectral imaging could concurrently image an object or a scene via hundreds or thousands of narrow bands with a spectral range that covers the various visible and infrared bands, has faced a growing interest in multifarious fields such as mineral exploration, military reconnaissance, agricultural analysis, etc. (Ravel, et al., 2018, Zhang et al., 2019). Hyperspectral imaging, also known as imaging spectroscopy.

Hyperspectral sensors provide spectrally rich information. However, low spatial resolution can especially be seen in data obtained from satellite platforms. Due to the low spatial resolution, the pixels in the hyperspectral images may contain more than one substance (Keshava and Mustard, 2002). For this reason, spectral unmixing aims at decomposing the measured mixed pixels into a collection of pure spectra, called endmembers, and into their corresponding proportions, called abundances. Spectral unmixing methods can be modeled as linear and nonlinear models (Keshava and Mustard, 2002; Bioucas-Dias et al., 2012; Heylen et al., 2014). The Linear Mixing Model (LMM) assumes that the mixed pixels are the sum of endmembers weighted by their corresponding abundances. That is, LMM assumes that the light coming to the hyperspectral sensor is proportional to the surface areas of the objects. It does not take into account the multiple scattering caused by environmental influences. The non-linear models, on the other hand, take into account the interaction between materials and try to find solutions to the mixing problem. The non-linear models often require prior knowledge of the physical properties of scene materials (Iordache et al., 2011). The computable traceability and flexibility of the linear models are the reasons for preference over the non-linear models.

A variety of spectral mixing techniques using LMM are based on convex geometry methods. Convex geometrybased approaches consider that the data is distributed in a simplex and most of these methods assume that some pixels in the data contain pure pixels. Vertex Component Analysis (Nascimento and Dias, 2005), Pixel Purity Index (Boardman et all., 1995) and N-Finder (Winter, 1999) are among the most popular methods assume that the data contains pure pixels. These methods aim to find the purest pixels in the datasets. However, since most of the datasets do not contain pure pixels, the use of these methods may not always be suitable for real problems. The second approach of convex-based methods is to find the smallest volume of simplex that surrounds all data. 
Iterated Constrained Endmembers (ICE) (Berman et al., 2004), Sparsity - Promoting ICE (SPICE) (Zare and Gader, 2007), its extended version (SPICEE) (Yuksel et al., 2016) and Minimum Volume Simplex Analysis (MVSA) (Li and Bioucas-Dias, 2008) are some methods of obtaining the endmembers from the datasets. If the data is too heavily mixed, these methods could extract virtual endmembers with no physical meaning (Iordache et al., 2011; Akhtar et al., 2015). To overcome the deficiencies of the convex geometry-based methods, the spectral mixing problem has been addressed by semisupervised unmixing approaches. These methods use spectral libraries containing material signatures collected by the spectrometers in the laboratory. The so-called sparse regression techniques assume that mixed pixels are expressed as a combination of only a few spectral signatures in a library (Iordache et al., 2011; Iordache et al., 2014; Akhtar et al., 2015). Sparse regression algorithms including Orthogonal Matching Pursuit (OMP), Basis Pursuit (BP), BP denoising (BPDN) are examined in (Iordache et al., 2011) for unmixing of hyperspectral data. Furthermore, some sparse unmixing methods have been developed by making use of the spatial information (Iordache et al., 2012) and subspace nature (Iordache et al., 2014; Shi et al., 2014) of the hyperspectral data. The sparse unmixing methods are generally based on convex relaxation of the problem. However, there are also several Greedy Approaches (GAs) that try to find an approximate solution to the $l_{0}$ problem directly. The GAs have the advantage of low computational complexity in solving the optimization problem (Iordache et al., 2011; Shi et al., 2014; Akhtar et al., 2015). However, the performance of GAs is influenced by the high correlation between the spectra of materials frequently occurring in spectral libraries.

In summary, the semi-supervised unmixing methods provide a sparse approximation solution with two different types of algorithms. These are the GAs and convex relaxation based versions. All these methods basically utilize LMM to characterize the mixed hyperspectral data. In this paper, we demonstrate that the bound-constrained least-squares, also an $l_{1}$ relaxationbased method that is implemented in CVX optimization software, can produce a sparse solution for mixing problems. To evaluate its unmixing performance, we compare the bound-constrained least-squares method with some convex relaxation-based sparse regression methods on a synthetic dataset.

\section{Materials and Methods}

Sparse regression techniques are derived from the sparse representation theory. Thus, the fundamentals of sparse representation theory are described in detail in this chapter. The sparse representation theory mainly deals with Compressed Sensing (CS) which is one of the popular topics in recent years. CS is used in a wide range of applications including signal processing; image processing, machine learning and computer vision (Zhang et al., 2015). In sparse modeling, a signal $x \in \mathbb{R}^{n}$ is modeled as a linear combination of the dictionary
$\mathbf{D} \in \mathbb{R}^{n x m}$ and the sparse representation vector $\gamma \in \mathbb{R}^{m}$ which has a few numbers of nonzero inputs.

The system model of sparse representation is shown in Figure 1. The main part of the model is the dictionary $\mathbf{D}$ which is also called the measurement matrix. The dictionary $\mathbf{D}$ consists of total $m$ atoms and the atoms are represented $\left\{d_{j}\right\}_{j=1}^{m}$ in the dictionary. The sparse representation vector $\gamma$ is a sparse vector with a few $(L$ entries) number of nonzero entries. For instance, the sparsity degree of vector $\gamma$ is $L=3$ in given model in Figure 1. The sparse representation of signal $x$ consists of a linear combination of dictionary atoms corresponding to the non-zero elements of the sparse representation vector $\gamma$.

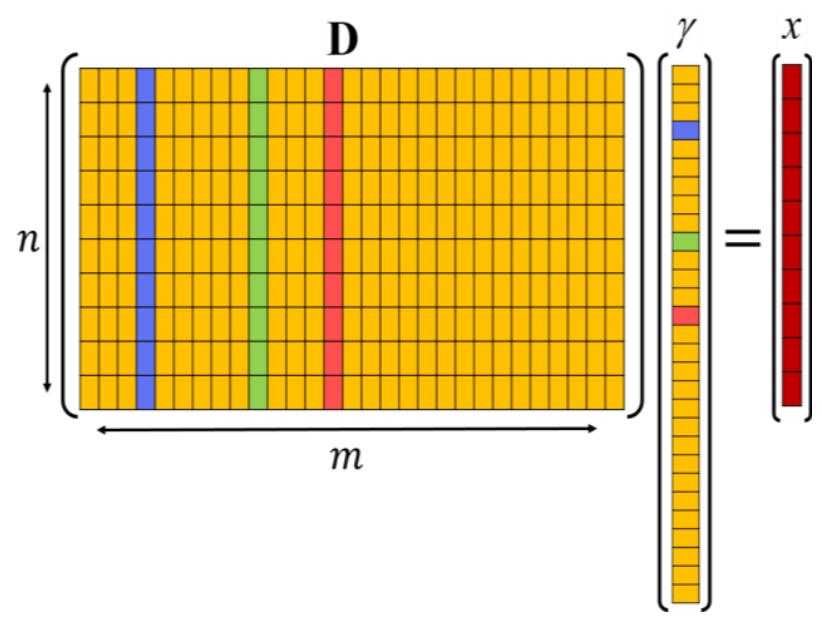

Fig. 1. The sparse representation model. The model can be expressed as $\mathbf{D} \gamma=x$.

The dictionary $\mathbf{D}$ is created with $(m>>n)$ in most cases. For instance, Figure 2 shows 256 different atoms (a patch of size $8 \times 8$ pixels) of the same size that build up the dictionary D. Each patch of the dictionary atoms should be vectorized for building the dictionary. For the example of Figure 2, the atoms consist of a vector of length $n=64$. Dictionaries created for cases where the total number of atoms is greater than the atoms size ( $m=$ $256 \gg n=64$ ) are called over-complete dictionaries. The mathematical expression of the sparse modeling is as follows:

$$
x=\mathbf{D} \gamma
$$

Equation (1) is essentially an under-determined linear system equation. The sparse vector $\gamma$ can be found by the pseudo-inverse if the dictionary $\mathbf{D}$ and the measurement vector $x$ are known. However, the pseudo-inverse solution is not unique and leads to an intense solution. In this case, it is not possible to ensure that very few entries of the sparse representation vector $\gamma$ have non-zeros. The sparse representation solution can be achieved by adding the constraint of minimizing $l_{0}$ norm to (1) (Elad, 2010). The equation of the sparse modeling becomes the optimization problem given in (2) by adding the $l_{0}$ norm.

$$
\min _{\gamma}\|\gamma\|_{0} \text { s.t } x=\mathbf{D} \gamma
$$


In (2), $\|\gamma\|_{0}$ represents $l_{0}$ norm of the sparse vector $\gamma$. The $l_{0}$ norm, used as a measure of sparsity, gives the number of non-zero entries in a vector.

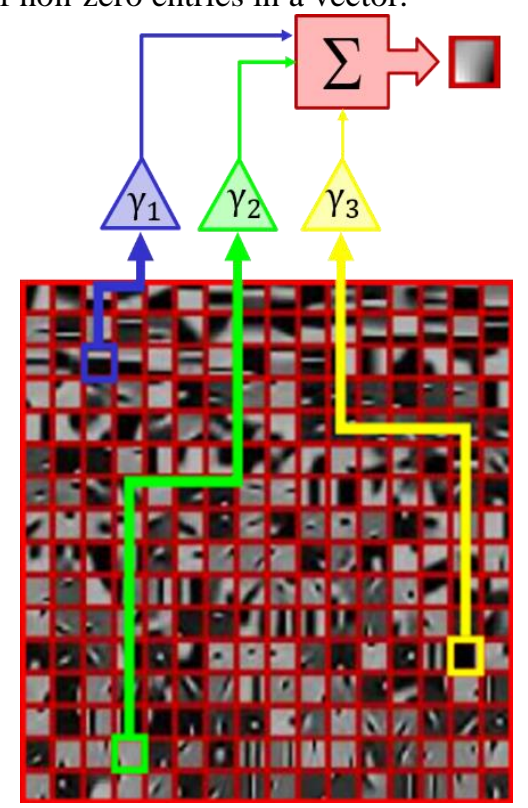

Fig. 2. The composition of an image patch using three building blocks from the dictionary (Papyan et al., 2018).

The problem given in (2) aims to produce the signal $x$ as a linear combination of a few atoms from the dictionary D. However, finding the dictionary atoms to meet the conditions in (2) may require a high computational cost. For instance; imagine a signal $x$ composed of 15 atoms of a dictionary containing 2000 atoms. It may be necessary to consider the probability of 15 of the 2000 atoms, which is about $2.4 \mathrm{e} 37$ probabilities. Even if each operation takes $1 \mathrm{ps}$, it will require billions of years to complete this task. This circumstance is expressed as NP-Hard (Non-deterministic Polynomial-time) problem (Papyan et al., 2018). Considering the NP-Hard problem, we are trying to find approximate solutions to the problem given by (2).

There are various methods in the literature that find approximate solutions. Matching Pursuit (Mallat and Zhang, 1993; Zhang et al., 2015), Orthogonal Matching Pursuit (Pati et al., 1993; Elad, 2010; Zhang et al., 2015), Basis Pursuit (Chen et al., 2001; Zhang et al., 2015) are most known algorithms. These methods are given in the subsections, respectively.

\section{Matching Pursuit}

Matching Pursuit (MP) is an iterative greedy method. It aims to find a single dictionary atom that provides a sparse approach to signal $x$ in each iteration. The process of MP is summarized in two steps:

-Selection of atoms from the dictionary

The first step is to find the atom that best matches with the signal. The dictionary atoms are normalized before this process. The best matching is determined by taking the inner product of the signal and the dictionary atoms. The purpose of this process is to find the dictionary atom that is most similar to the signal. The dictionary atom has the largest inner product is selected as the best matching atom. The mathematical expression of these operations is given in (3).

$$
d^{k}=\max _{\left\{d_{j}\right\}}\left|<r^{k-1}, d_{j}>\right|, \quad 1 \leq \mathrm{j} \leq m \quad \text { (Eq. 3) }
$$

where $d_{j}$ denotes $j^{\text {th }}$ atom of the dictionary, $r$ denotes residual signal and $\langle\cdot\rangle$ denotes inner product operation. Initially, residual set to measurement signal, $r^{0}=x$. The scalar value obtained from the inner product, $\quad \gamma=<r^{k-1}, d^{k}>$ is calculated as the coefficient for the determined $d^{k}$ atom. When the algorithm ends, the pair $\left(d^{k}, \gamma^{k}\right)$ is kept in memory to compose the signal.

- Update the residual signal

The weighted value of the atom found in the first step is subtracted from the signal to give a residual signal. The first step is repeated over the obtained residual signal. This process continues until a certain stop condition is satisfied. As a stopping condition, it is usually defined as the norm of the residual signal falling below a certain threshold value. Finally, using the $\left(d^{k}, \gamma^{k}\right)$ pairs stored in memory, the approximate $\hat{x}$ signal is obtained by the following mathematical expression.

$$
\hat{x}=\sum_{j=1}^{k} \gamma^{j} d^{j}
$$

MP is a simple and practical method. However, since all dictionary atoms are scanned at each iteration, it has a processing cost.

\section{Orthogonal Matching Pursuit}

Orthogonal Matching Pursuit (OMP) is a greedy algorithm aimed at finding an approximate solution to sparse problems such as MP. OMP is also an iterative algorithm. The main steps of OMP are summarized in Algorithm 1 (Zhang et al., 2015). OMP uses a procedure similar to MP. In contrast, OMP projects the residual signal in each iteration into a subspace spanned by all selected atoms until that iteration. This difference occurs after Step 2 given in Algorithm 1.

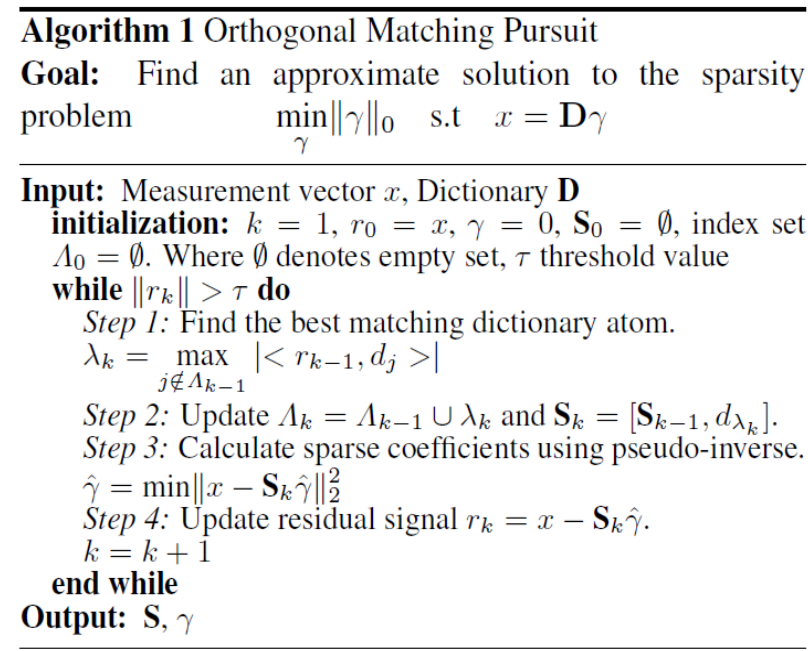




\section{Basis Pursuit}

Unlike the greedy algorithms, the Basis Pursuit (BP) method uses the $l_{l}$ norm instead of the $l_{0}$ norm in (2) to calculate the sparse representation vector. This alteration provides relaxation on (2) and therefore, such methods are called relaxation methods. $\mathrm{BP}$ tries to solve the optimization problem given by the mathematical expression in (5).

$$
\min _{\gamma}\|\gamma\|_{1} \text { s.t } x=\mathbf{D} \gamma
$$

Equation (5) is an equation converted to a convex optimization problem and can be solved by linear programming algorithms. The noise added form of (5) is also written as in (6):

$$
\min _{\gamma}\|x-\mathbf{D} \gamma\|_{2}^{2}+\lambda\|\gamma\|_{1}
$$

where $\lambda$ is a penalty term that increases the sparsity on non-zero elements. The first term of (Eq. 6) is always greater than zero. The second term is the sum of the absolute value of the entries of the vector $\gamma$ which will always be greater than zero. As the penalty term $\lambda$ becomes larger, since the second term becomes more dominant than the first term, the minimization of the problem will be possible by forcing $\gamma$ values to zero. Thus, the sparse representation of vector $\gamma$ will be provided.

\section{Linear Mixing Model}

Hyperspectral mixing problem which is considered as a sparse approximation problem can be formulated with Linear Mixing Model. LMM assumes that the spectral response in a spectral band consists of a linear combination of the endmembers. Mathematically expressed as follows:

$$
x_{i}=\sum_{j=1}^{m} e_{i j} p_{j}+\varepsilon_{j}
$$

where $x_{i}$ is the spectral reflection measured in the $i^{\text {th }}$ spectral band, $m$ is the total number of endmember, $e_{i j}$ is the reflection value of the $j^{\text {th }}$ endmember in the $i^{\text {th }}$ spectral band, $p_{j}$ is the abundance value of the $j^{\text {th }}$ endmember and $\varepsilon_{j}$ is the measurement error. Assuming that the hyperspectral data cube contains $n$ spectral channels, our model can be written in compact matrix form as follows:

$$
x=\mathbf{E} p+\varepsilon
$$

where, $x \in \mathbb{R}^{n}$ represents the measured reflection at one pixel, $\mathbf{E} \in \mathbb{R}^{n x m}$ is a matrix containing endmembers, $p \in \mathbb{R}^{m}$ is the abundance vector and $\varepsilon \in \mathbb{R}^{n}$ is the error vector. The LMM has two constraints for abundance values (Keshava and Mustard, 2002; Bioucas-Dias et al., 2012). The first constrain is the abundance values are not negative, and the second is summation of abundance values must be equal to 1 .

$$
p>0
$$

$$
\mathbf{1}^{T} p=1
$$

These constraints are imposed because LMM assumes that the light coming to the hyperspectral sensor is proportional to the surface areas of the materials.

\section{Sparse Spectral Unmixing}

Sparse approximation methods can also be used for spectral unmixing if the LMM is reconstructed. Assuming that the $\mathrm{D}$ dictionary to be created with libraries including the spectra of the materials contains all the endmember signatures in mixed pixels, the mixed signal $x$ is expressed as follows:

$$
x=\mathbf{D} \gamma+\varepsilon
$$

where $\gamma \in \mathbb{R}^{m}$ is a sparse abundance vector with very few $(p \ll m)$ non-zero entries. When the constraints of the LMM are added to (11), the solution of the optimization problem is as follows:

$\min _{\gamma}\|\gamma\|_{0}$ s.t $\|\mathbf{D} \gamma-x\|_{2} \leq \eta, \gamma>0, \mathbf{1}^{T} \gamma=1$ (Eq. 12)

where $\eta$ denotes the error tolerance. Equation (12) can be solved with greedy sparse approximation algorithms (Akhtar et al., 2015; Iordache et al., 2011; Toker and Yuksel, 2018). The relaxation of the minimization problem has also been widely studied for the past few years. Most of these methods try to solve the sparse mixing problem by adding the constraints of the LMM to (6). Dias and Figueiredo have developed an algorithm named as the Spectral Unmixing by Variable Splitting and Augmented Lagrangian (SUNSAL) (Bioucas-Dias and Figueiredo, 2010) to solve the problem given in (6). In the same study, the constrained version of SUnSAL (CSUnSAL) algorithm was also developed to solve the hyperspectral mixing problem. Both SUNSAL and CSUnSAL use the Alternating Direction Method of Multipliers (ADMM) for sparse unmixing. An improved version of SUnSAL called CLSUnSAL (CoLlaborative SUnSAL) tries to unmix all mixed pixels simultaneously (Iordache et al., 2014).

\section{Data Generation for Sparse Spectral Unmixing}

In this study, firstly, a dictionary $\mathbf{D}$ is created containing the spectral signatures of the materials. For this purpose, USGS spectral libraries which contain spectral signatures of hundreds of materials with different properties such as plant species, minerals, organic compounds, soil and rock, man-made materials are used (Kokaly et al., 2017). Spectral signatures consist of 118 spectral bands (some bands are removed) in $0.4-2.45$ $\mu \mathrm{m}$ range. The USGS library contains over 2000 spectral signatures. In the mixing problem, the excessive similarity of the material signatures in a pixel makes the spectral unmixing process difficult. Therefore, different material signatures have been tried to be selected as much as possible to build the dictionary. For this purpose, the mutual coherence metric which is mathematically expressed in (13) is used. 


$$
\mu(\mathbf{D})=\max _{i, j ; i \neq j} \frac{\left|d_{i}^{T} d_{j}\right|}{\left\|d_{i}\right\|_{2}\left\|d_{j}\right\|_{2}}
$$

The spectra of materials are chosen such that their mutual coherence $\mu$ is 0.997 and the dictionary $\mathbf{D} \in$ $\mathbb{R}^{188 \times 454}$ is created. Five different spectra are randomly selected from the dictionary and 100 mixed pixels are

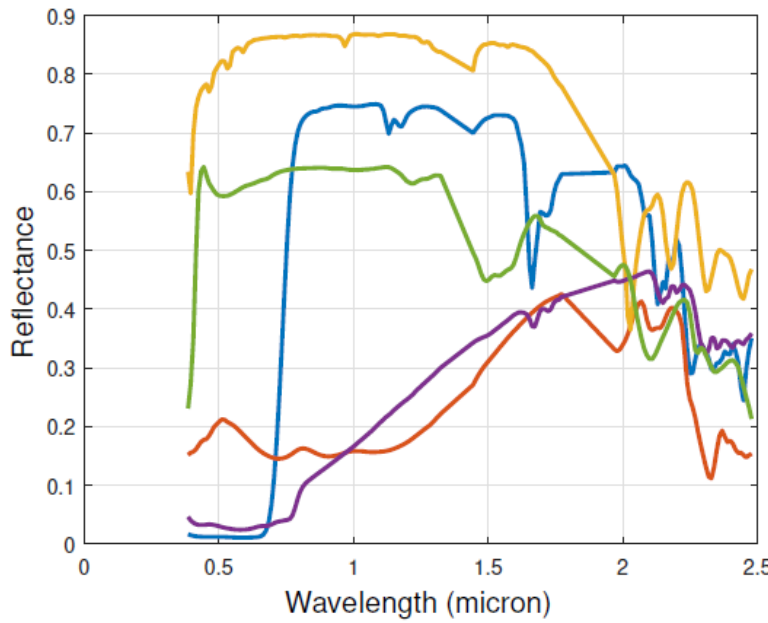

(a) generated using the LMM model given in (8). Abundances are generated using the Dirichlet distribution such that they sum to 1 in each pixel. The selected material signatures from the dictionary and spectra of a mixed pixel are shown in Figure $3 a$ and Figure $3 b$, respectively.

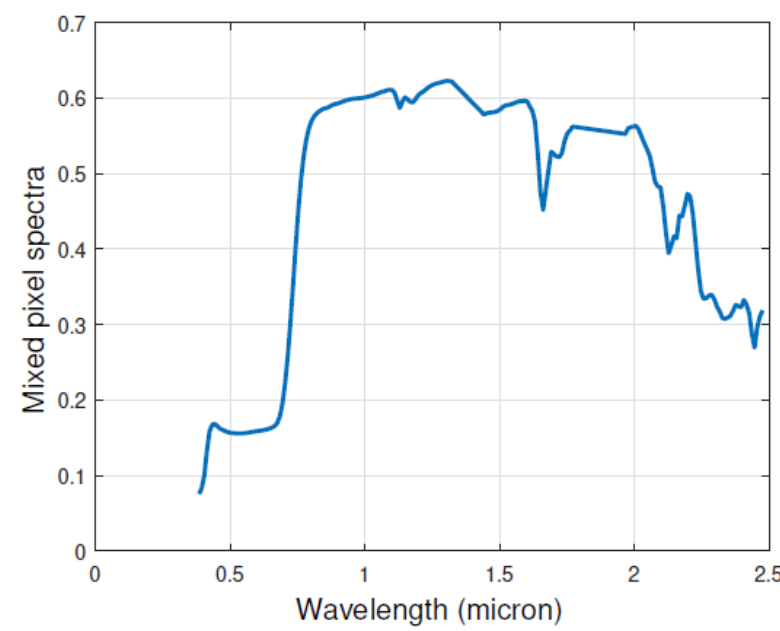

(b)

Fig. 3. (a) Five different material signatures (b) a mixed pixel spectra obtained as a combination of five selected

signatures from
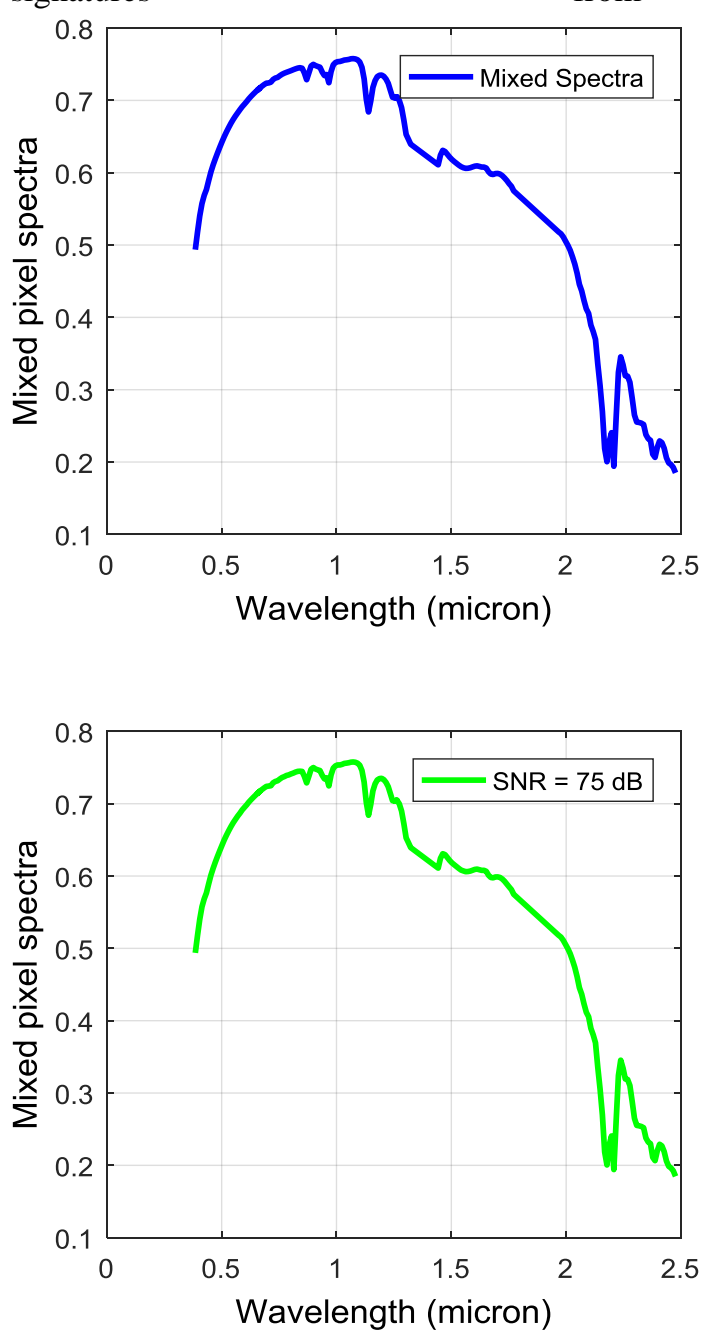

USGS
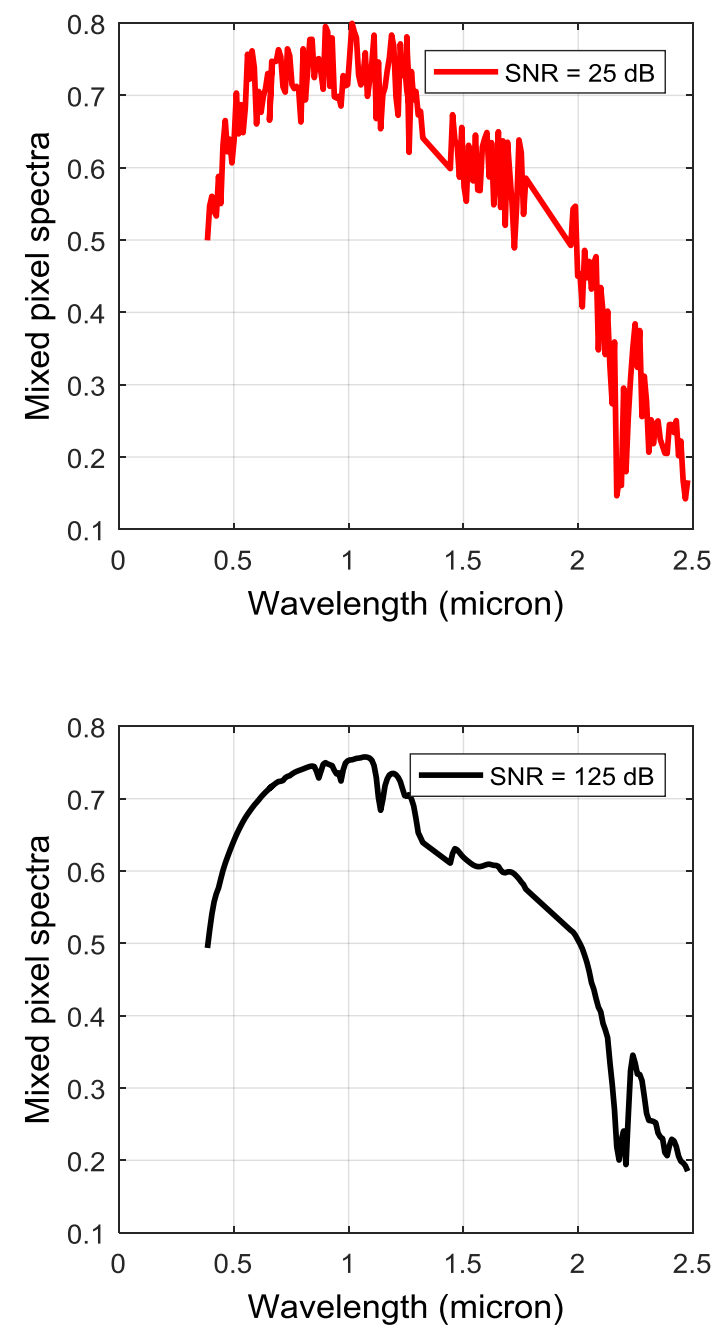

Fig. 4. Example of white noise: In the given mixed spectra, white noise is added to obtain different levels of SNR. 


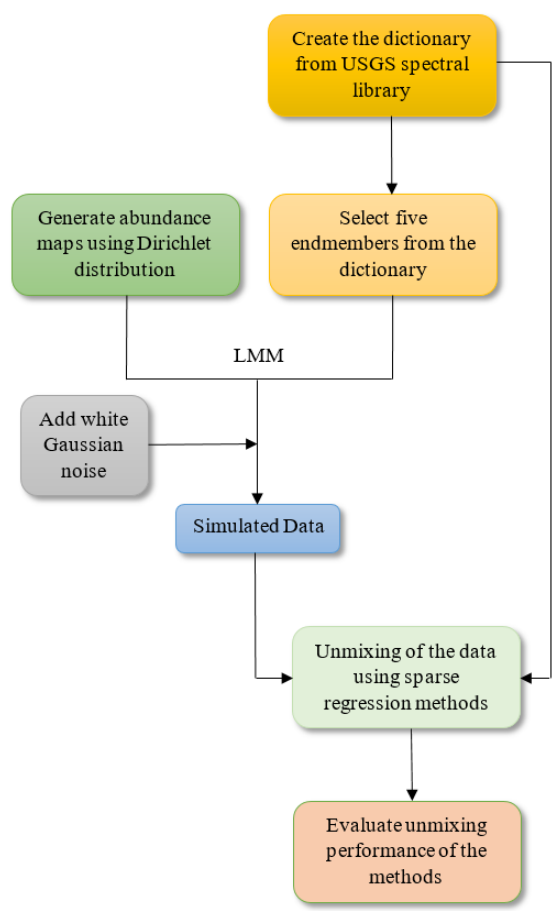

Fig. 5. Flowchart of the study.

The noise is added to the mixed simulated data at three different levels of SNR $=25 \mathrm{~dB}, \mathrm{SNR}=75 \mathrm{~dB}$ and SNR $=125 \mathrm{~dB}$. Figure 4 shows a randomly selected mixed pixel spectra from the simulated dataset and its noise added spectra with different SNR levels. The general flowchart of the simulated dataset generation and unmixing procedure is shown in Figure 5.

\section{Experimental Results}

In this study, CVX - MATLAB software for convex programming (Grant and Boyd 2014) is used for sparse unmixing of the produced synthetic data. Abundances should have two constraints: (i) they should be positive as they indicate the ratio of some material to exist, and (ii) they should sum to one as we consider the linear mixing model. By adding these two constraints of abundances to Eq. 6, the equation given below is obtained.

$$
\min _{\gamma}\|\mathbf{D} \gamma-x\|_{2}^{2}+\lambda\|\gamma\|_{1}, \gamma>0, \mathbf{1}^{T} \gamma=1
$$

The bound-constrained least squares (BCLS) method is used in the CVX for the solution of (14). In addition, the performance of the sparse regression of BCLS is compared with sparse unmixing methods and the results are given in Table 1. The method represented by LS in Table 1 is the least squares method that occurs when $\lambda=0$. The Root Mean Square Error (RMSE), Signal to Reconstruction Error (SRE) which is mathematically expressed in (15) and the time spent by the algorithms in unmixing 100 mixed pixels is used as comparison criterions.

$$
\operatorname{SNR}(\mathrm{dB})=10 \log _{10}\left(\frac{\|x\|^{2}}{\|x-\hat{x}\|^{2}}\right)
$$

It can be seen from Table 1 that BCLS produces very close results to the actual abundances, especially when the noise level is low $(\mathrm{SNR}=125 \mathrm{~dB})$. However, due to the pixel-by-pixel analysis of the mixing problem, BCLS spends much more time than other methods. Hinton diagrams are used to visualize which material signatures are found in the library $\mathbf{D}$ and the proportions of these found material signatures. Hinton diagrams are often used in the field of artificial neural networks to visualize matrices. The area covered by the cells in the diagram is proportional to the matrix values. For $\mathrm{SNR}=25 \mathrm{~dB}$, the sparse representation vectors obtained as a result of sparse regression methods are shown in Figure 6. In each of the graphs in Figure 6, the $x$-axis represents pixels and the $y$-axis shows which endmembers in the dictionary are found in mixed pixels. Hinton diagrams for the mixed data having SNR of $125 \mathrm{~dB}$ are given in Figure 7. It is seen from the Hinton diagrams that LS give quite misleading results for two different noise levels. Since the LS did not impose any constraints on abundances, it produced a dense solution rather than a sparse solution. In Figure 6, it is seen that sparse regression methods can clearly find the coefficients that correspond to actual endmembers. However, it is also seen that the mixed pixels contain other endmembers. That is, the regression methods have not been able to reduce the sparse representation vector values to zero sufficiently. In the graphs shown in Figure 7, while the noise level is low, the sparse regression methods except for LS have successfully found which endmembers are mixed in the pixels. Furthermore, they are able to estimate the abundances of these endmembers with very high accuracy.

\section{Discussion and Conclusion}

In this study, the results on the generated dataset have shown that Sparse unmixing is a powerful technique to solve the spectral mixing problem. It has been shown that sparse regression methods derived from BP can successfully solve the spectral mixing. The boundconstrained least-squares method yields similar results with these methods. However, it is a time-consuming procedure when compared to BP-based methods because it calculates the abundances pixel by pixel. But, the implementation of the bound-constrained least-squares method can be developed in parallel computing environments for faster unmixing processing.

Sparse regression methods use dictionary atoms created by spectral libraries to analyze mixed pixels. Although the number and variety of material signatures in spectral libraries increase gradually, a mixed pixel may contain an endmember that is not present in the library. In this paper, experiments are not performed for the cases where the endmembers in the mixed pixels are not included in 
Table 1. Comparison of the unmixing performance of sparse regression methods.

\begin{tabular}{lrrr||rrr||rrr} 
& \multicolumn{3}{c}{ SNR = $25 \mathrm{~dB}$} & \multicolumn{3}{c||}{ SNR = 75 dB } & \multicolumn{3}{c}{ SNR = $125 \mathrm{~dB}$} \\
\cline { 2 - 9 } & RMSE & SRE(dB) & Time $(\mathrm{sec})$ & RMSE & SRE(dB) & Time $(\mathrm{sec})$ & RMSE & SRE(dB) & Time $(\mathrm{sec})$ \\
\hline LS & 592.7 & -43.33 & $\mathbf{0 . 0 1}$ & 3.57 & 0.61 & $\mathbf{0 . 0 1}$ & 3.12 & 3.43 & $\mathbf{0 . 0 1}$ \\
SUnSAL & 1.92 & 6.71 & 1.96 & 1.76 & 10.30 & 0.83 & 0.08 & 36.88 & 0.43 \\
CSUnSAL & 1.43 & 10.09 & 1.62 & 0.10 & 34.18 & 0.73 & 0.01 & 54.26 & 0.45 \\
CLSUnSAL & $\mathbf{1 . 4 2}$ & $\mathbf{1 0 . 1 8}$ & 2.18 & $\mathbf{0 . 0 3}$ & $\mathbf{4 4 . 1 9}$ & 2.09 & 0.002 & 66.11 & 1.49 \\
BCLS & 1.43 & 10.09 & 103.75 & 0.08 & 35.97 & 101.09 & $\mathbf{0 . 0 0 0 1}$ & $\mathbf{9 0 . 7 1}$ & 119.27
\end{tabular}

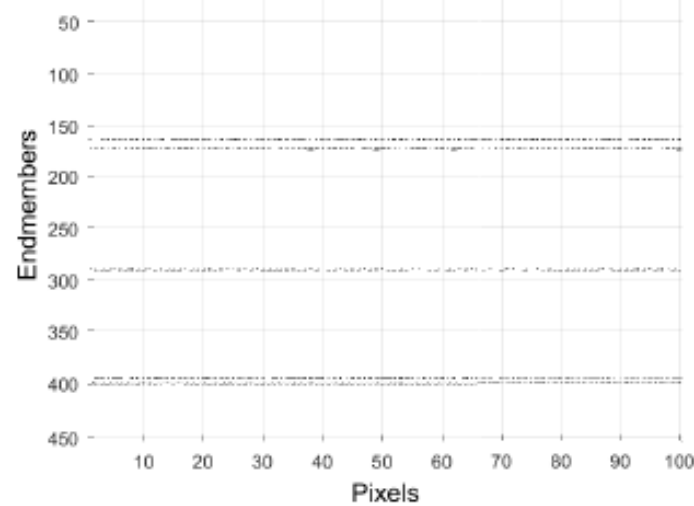

(a) Ground-truth

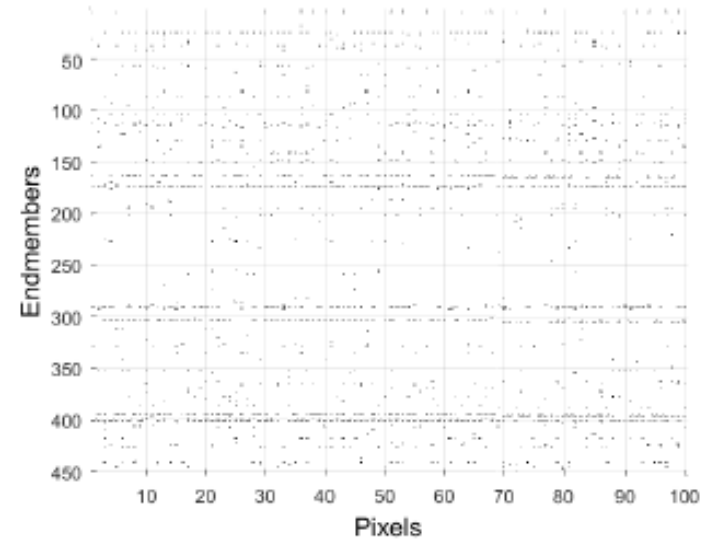

(c) SUnSAL

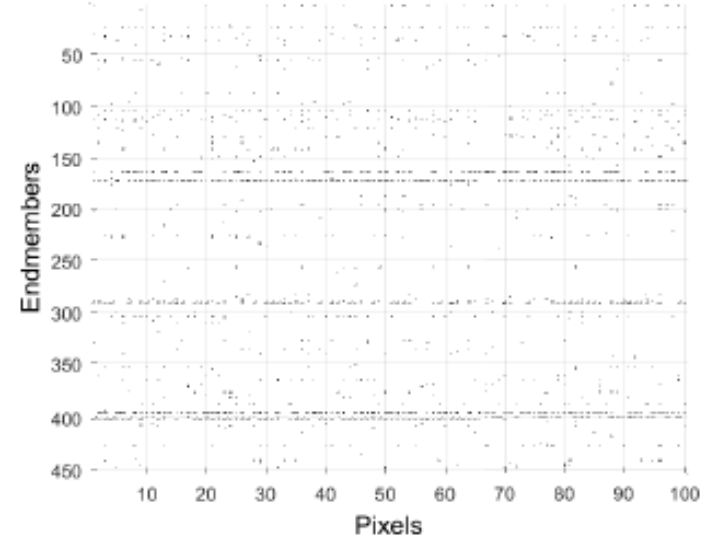

(e) CLSUnSAL

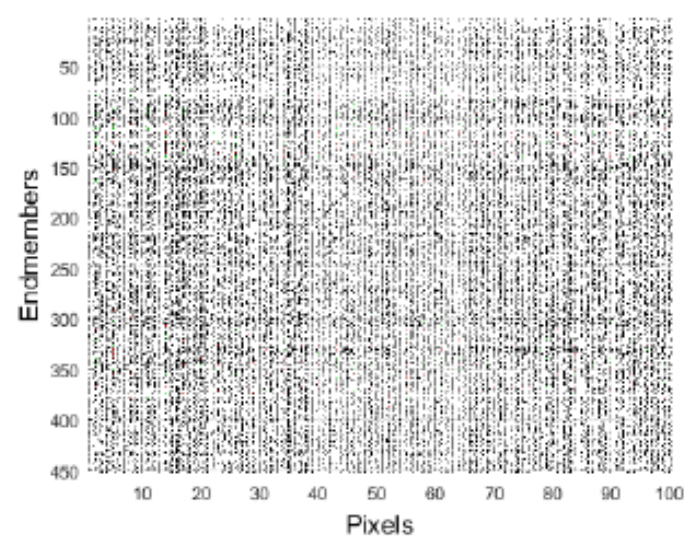

(b) LS

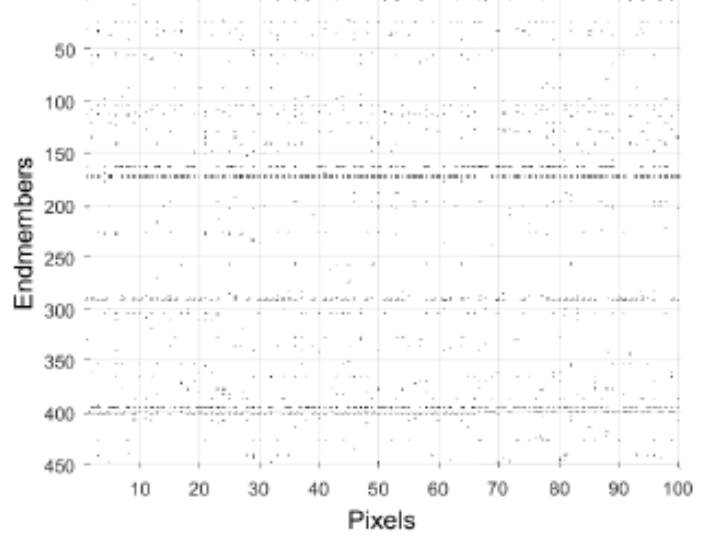

(d) CSUnSAL

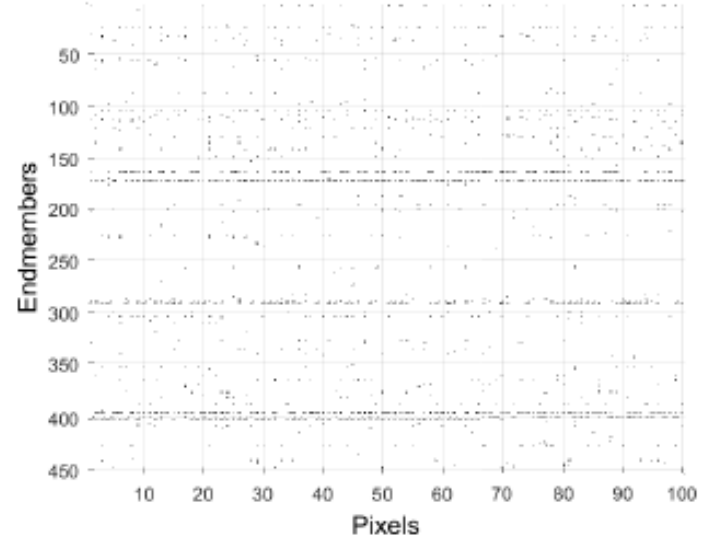

(f) BCLS

Fig. 6. Estimated abundances of each endmember in the dictionary D. (The results are for the mixed data having SNR of $25 \mathrm{~dB})$ 


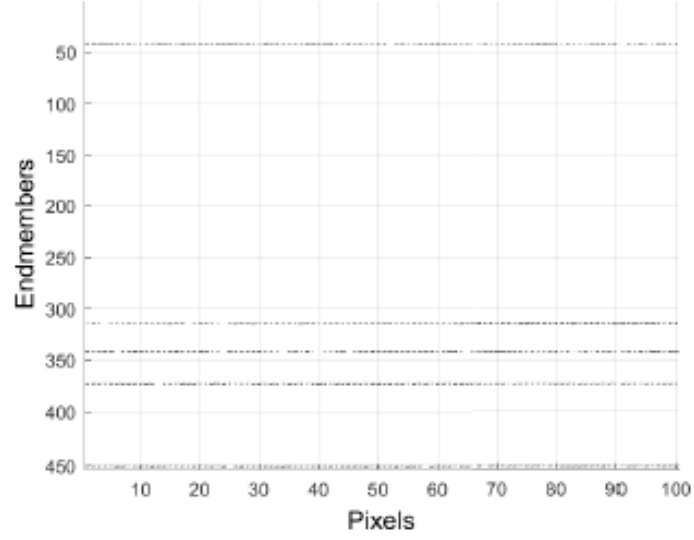

(a) Ground-truth

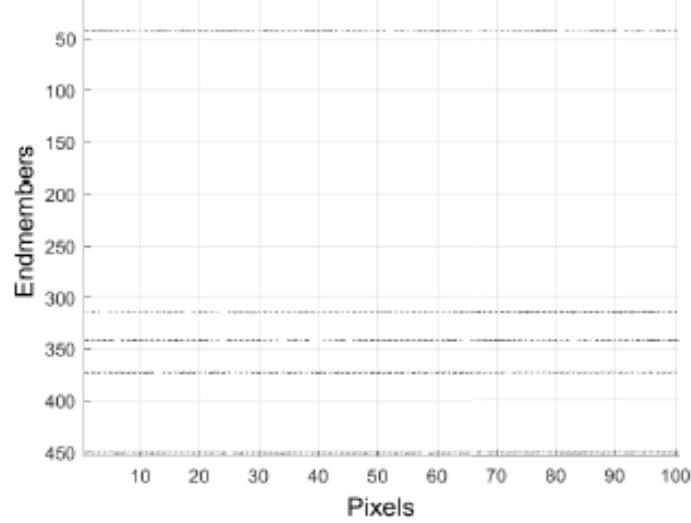

(c) SUnSAL

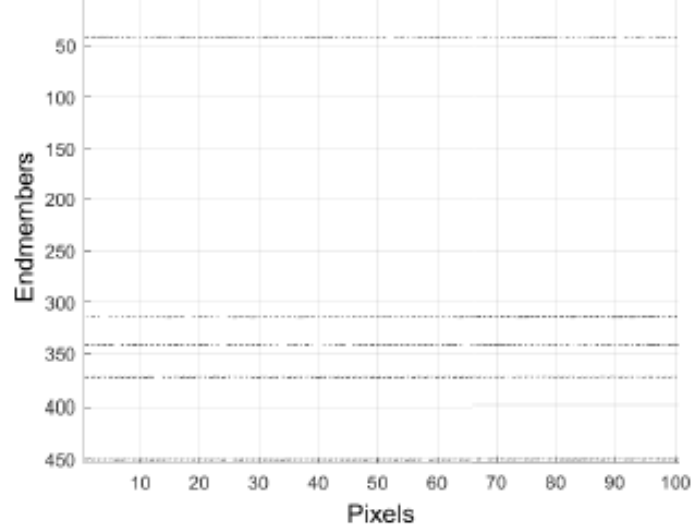

(e) CLSUnSAL

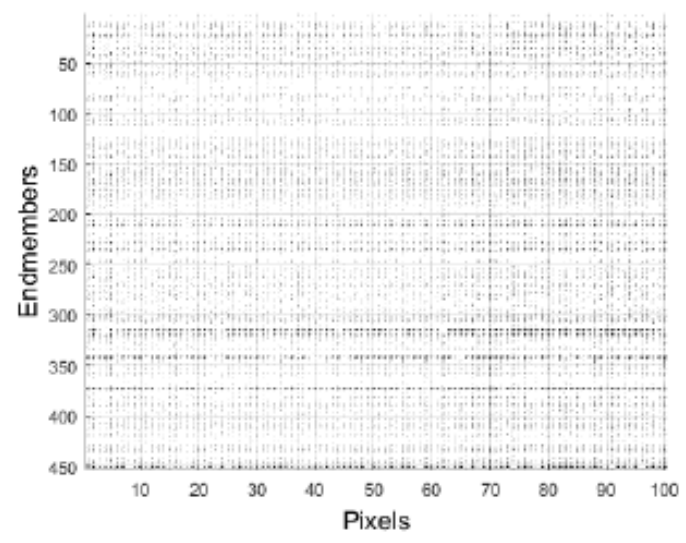

(b) LS

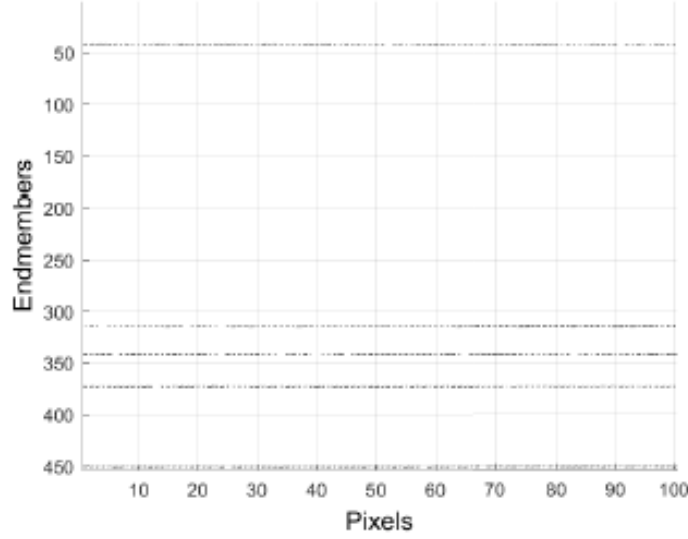

(d) CSUnSAL

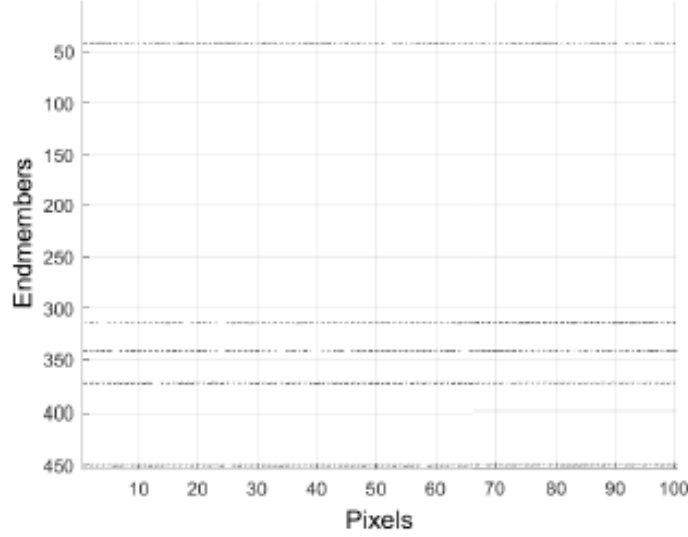

(f) BCLS

Fig. 7. Estimated abundances of each endmember in the dictionary D. (The results are for the mixed data having SNR of $125 \mathrm{~dB}$ )

the dictionary atoms. However, if the target material signature in the mixed pixel is not one of the dictionary atoms, it can be predicted that sparse regression methods will select the spectral signature of a highly similar endmember among dictionary atoms as the target signature.

\section{References}

Akhtar, N., Shafait, F., Mian, A. (2015). Futuristic greedy approach to sparse unmixing of hyperspectral data. IEEE Transactions on Geoscience and Remote Sensing, 53(4), 2157-2174.

Berman, M., Kiiveri, H., Lagerstrom, R., Ernst, A., Dunne, R., Huntington, J. F. (2004). ICE: A statistical approach to identifying endmembers in hyperspectral images. IEEE Transactions on Geoscience and Remote Sensing, 42(10), 2085-2095. 
Bioucas-Dias, J. M., Figueiredo, M. A. (2010, June). Alternating direction algorithms for constrained sparse regression: Application to hyperspectral unmixing. In 2010 2nd Workshop on Hyperspectral Image and Signal Processing: Evolution in Remote Sensing (pp. 1-4). IEEE.

Bioucas-Dias, J. M., Plaza, A., Dobigeon, N., Parente, M., Du, Q., Gader, P., Chanussot, J. (2012). Hyperspectral unmixing overview: Geometrical, statistical, and sparse regression-based approaches. IEEE Journal of Selected Topics in Applied Earth Observations and Remote Sensing 5(2), 354-379.

Boardman, J. W., Kruse, F. A., Green, R. O. (1995). Mapping target signatures via partial unmixing of AVIRIS data.

Chen, S. S., Donoho, D. L., Saunders, M. A. (2001). Atomic decomposition by basis pursuit. SIAM review, 43(1), 129-159.

Elad, M. (2010). Sparse and redundant representations: from theory to applications in signal and image processing. Springer Science \& Business Media.

Grant, M., Boyd, S. (2014). CVX: Matlab software for disciplined convex programming, version 2.1.

Heylen, R., Parente, M., Gader, P. (2014). A review of nonlinear hyperspectral unmixing methods. IEEE Journal of Selected Topics in Applied Earth Observations and Remote Sensing, 7(6), 1844-186

Iordache, M. D., Bioucas-Dias, J. M., \& Plaza, A. (2012). Total variation spatial regularization for sparse hyperspectral unmixing. IEEE Transactions on Geoscience and Remote Sensing, 50(11), 44844502.

Iordache, M. D., Bioucas-Dias, J. M., Plaza, A. (2011). Sparse unmixing of hyperspectral data. IEEE Transactions on Geoscience and Remote Sensing, 49(6), 2014-2039.

Iordache, Marian-Daniel, Bioucas-Dias, Jose M, Plaza, Antonio, 2014. Collaborative sparse regression for hyperspectral unmixing. IEEE Transactions on Geoscience and Remote Sensing, 52, 341--354.

Keshava, N., \& Mustard, J. F. (2002). Spectral unmixing. IEEE Signal Processing Magazine, 19(1), 44-57.

Kokaly, Raymond F, Clark, Roger N, Swayze, Gregg A, Livo, K Eric, Hoefen, Todd M, Pearson, Neil C, Wise, Richard A, Benzel, William M, Lowers, Heather A, Driscoll, Rhonda L et al., (2017). Usgs spectral library version 7. Technical report, US Geological Survey.

Kucuk, S, (2015). Target detection from long-wave infrared hyperspectral images. (Master's thesis). Hacettepe University, Ankara, Türkiye.

Li, J., Bioucas-Dias, J. M. (2008, July). Minimum volume simplex analysis: A fast algorithm to unmix hyperspectral data. In IGARSS 2008-2008 IEEE
International Geoscience and Remote Sensing Symposium (Vol. 3, pp. III-250). IEEE.

Mallat, S. G., Zhang, Z. (1993). Matching pursuits with time-frequency dictionaries. IEEE Transactions on Signal Processing, 41(12), 3397-3415.

Nascimento, J. M., Dias, J. M. (2005). Vertex component analysis: A fast algorithm to unmix hyperspectral data. IEEE transactions on Geoscience and Remote Sensing, 43(4), 898-910.

Papyan, V., Romano, Y., Sulam, J., Elad, M. (2018). Theoretical foundations of deep learning via sparse representations: A multilayer sparse model and its connection to convolutional neural networks. IEEE Signal Processing Magazine, 35(4), 72-89.

Pati, Y. C., Rezaiifar, R., Krishnaprasad, P. S. (1993, November). Orthogonal matching pursuit: Recursive function approximation with applications to wavelet decomposition. In Proceedings of 27th Asilomar conference on signals, systems and computers (pp. 40-44). IEEE.

Ravel, S., Fossati, C., Bourennane, S. (2018). Spectral Unmixing of Hyperspectral Images in the Presence of Small Targets. Remote Sensing. 7(1), 10.18282/rs.v7i1.460.

Shi, Z., Tang, W., Duren, Z., \& Jiang, Z. (2014). Subspace matching pursuit for sparse unmixing of hyperspectral data. IEEE Transactions on Geoscience and Remote Sensing, 52(6), 3256-3274.

Toker, K. G., Yuksel, S. E. (2018, May). A greedy algorithm for sparse unmixing. In 2018 26th Signal Processing and Communications Applications Conference (SIU) (pp. 1-4). IEEE.

Winter, M. E. (1999, October). N-FINDR: An algorithm for fast autonomous spectral end-member determination in hyperspectral data. In Imaging Spectrometry $V$ (Vol. 3753, pp. 266-275). International Society for Optics and Photonics.

Yuksel, S. E., Kucuk, S., Gader, P. D. (2016). SPICEE: An Extension of SPICE for Sparse Endmember Estimation in Hyperspectral Imagery. IEEE Geoscience and Remote Sensing Letters, 13(12), 1910-1914.

Zare, A., Gader, P. (2007). Sparsity promoting iterated constrained endmember detection in hyperspectral imagery. IEEE Geoscience and Remote Sensing Letters, 4(3), 446-450.

Zhang, Z., Xu, Y., Yang, J., Li, X., Zhang, D. (2015). A survey of sparse representation: algorithms and applications. IEEE access, 3, 490-530.

Zhang, Z., Liao, S., Fang, H., Zhang, H., Wang, S. (2019). Sparse Hyperspectral Unmixing Using Spectral Library Adaptive Adjustment, IEEE Journal of Selected Topics In Applied Earth Observations and Remote Sensing, 1-15. DOI: 10.1109/JSTARS.2019.2939829 